\title{
Facial nerve paresis in the course of masked mastoiditis as a revelator of GPA
}

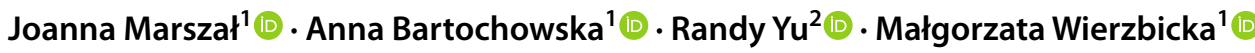

Received: 3 August 2021 / Accepted: 2 November 2021 / Published online: 19 November 2021

(c) The Author(s) 2021

\begin{abstract}
Purpose The aim of this study was to present a series of 6 patients with facial nerve palsy and masked mastoiditis which constituted as revelators of localized granulomatosis with polyangiitis (GPA) and to evaluate the utility of the ACR/EULAR 2017 provisional classification criteria for GPA in such cases.

Methods Study group included 58 patients with GPA. Cases with facial nerve palsy and masked mastoiditis were thoroughly analyzed.

Results The mean age of patients was 37 years. All manifested unilateral facial nerve palsy and hearing loss, while only 2 reported aural complaints suggesting inflammatory cause of the disease. All cases were qualified for surgical intervention. Intraoperative findings were similar: granulation tissue in tympanic cavity and/or pneumatic spaces of the mastoid process. Only $50 \%$ of histopathological results suggested vasculitis. In all cases, elevated levels of antineutrophil cytoplasmic antibodies (ANCA) against peroxidase 3 (PR3-ANCA) were determined. Two patients presented rapid progression of the disease and died within 1 week and 2 months, respectively. Four other patients manifested gradual improvement of hearing and facial nerve function after treatment.

Conclusion GPA should be included into differential diagnosis in all cases of persistent facial nerve palsy especially when otological symptoms coexist. Even localized GPA could be very aggressive, revelating generalized form of the disease. Rapid systemic treatment of GPA can protect hearing and facial nerve from permanent severe dysfunction. The ACR/EULAR 2017 provisional classification criteria for GPA seem to be valuable tool in diagnosing ENT patients with localized otological form of the disease.
\end{abstract}

Keywords GPA $\cdot$ Facial nerve palsy $\cdot$ Masked mastoiditis $\cdot$ c-ANCA

\section{Introduction}

Localized granulomatosis with polyangiitis (GPA), an autoimmune necrotizing small-vessel vasculitis, may affect ear, nose, larynx, and trachea [1]. Otologic involvement is quite common among ENT symptoms of the disease, with up to $50 \%$ of patients manifesting ear pathology [2-4]. The most common location of the otologic involvement is the middle ear--it presents as serous otitis media, chronic otitis media,

Anna Bartochowska

annabartochowska@gmail.com

1 Department of Otolaryngology, Head and Neck Surgery, Poznan University of Medical Sciences, 49 Przybyszewskiego Street, 60-355 Poznan, Poland

2 Graduate of Advanced MD Program, Poznan University of Medical Sciences, Poznan, Poland or chronic Eustachian tube dysfunction [5]. The second group of symptoms concerns inner ear and manifests as sensorineural hearing loss which is very often accompanied by tinnitus or vertigo [6,7]. Some cases of external otitis with atypical course were also reported [8]. Facial nerve palsy, usually associated with inflammation in middle ear spaces, is quite unique and can be observed in $8-10 \%$ of patients [5,9].

Masked mastoiditis is a rare subclinical complication of acute or chronic otitis media. The oligosymptomatic nature of the complication, often accompanied by other intracranial pathologies, causes great diagnostic difficulties. In the pathogenesis of this insidious clinical entity, the aditus ad antrum is often blocked by inflammatory mucosa, granulation tissue, or cholesteatoma [10]. Symptoms caused by persistent inflammation in mastoid air cells include pain localized deep in middle ear and retroauricular region, conductive hearing loss, and recurrent fever episodes. In most 
patients, no otoscopic changes or only thickening of tympanic membrane is observed. This oligosymptomatic nature often delays proper diagnosis. If mastoiditis is left untreated, it can lead to other complications, including facial nerve palsy [11]. While in classic inflammatory process, the main mechanism of paresis is the direct intoxication of the nerve by pathogens [12], the process in GPA involves the inflammation and occlusion of the "vasa nervorum" segment [13]. Some authors [5, 7] still support the theory of the facial nerve damage in the mechanism of compression by granulomatous lesions, and that hypothesis probably should also be considered in the palsy which occurs in the course of mastoiditis.

Although otological manifestations are quite common in GPA, unspecific clinical picture, including facial nerve paresis, may postpone appropriate diagnosis. Delayed treatment usually leads to progression to irreversible phase of the disease. Therefore, timely diagnosis of localized form is so important.

The purpose of this study was to present a series of 6 patients with facial nerve palsy and masked mastoiditis which constituted as revelators of GPA. We also aimed to evaluate the utility of the ACR/EULAR 2017 provisional classification criteria for GPA [14] in such sophisticated cases.

\section{Material and methods}

All patients with GPA treated in our department in 2008-2019 (58 altogether) were included into the analysis. We focused on those with facial nerve palsy and masked mastoiditis which were the first clinical symptoms of the disease. These cases were thoroughly analyzed. The following variables were collected: age, gender, symptoms, clinical picture, diagnostic parameters of GPA [level and type of antineutrophil cytoplasmic antibodies (c-ANCA)], auditory tests (subjective audiometry, tympanometry) values, result of high-resolution computed tomography (HRCT) of the temporal bone, method of treatment, intraoperative findings, histological results, and outcomes.

All data were evaluated according to 1990 criteria for the classification of Wegener's granulomatosis (now known as GPA) established by the American College of Rheumatology (ACR) [15] (Table 1) and 2017 ACR/EULAR provisional classification criteria for GPA presented at 2016 ACR session: New Classification Criteria for ANCA-associated Vasculitis: implications for clinical practice [14] (Table 2).

This study was approved by our institutional bioethics committee.

\section{Results}

Our study group consisted of 3 men and 3 women with a mean age of 37 years (range 31-43 years). All patients manifested unilateral facial nerve palsy and hearing loss (unilateral or bilateral, mixed type, mean hearing thresholds

Table 2 The ACR/EULAR 2017 provisional classification criteria for GPA [14]

\begin{tabular}{lr}
\hline Item & Score \\
\hline $\begin{array}{l}\text { Bloody nasal discharge, ulcers, crusting or sinonasal conges- } \\
\quad \text { tion }\end{array}$ & 3 \\
Nasal polyps & -4 \\
Hearing loss or reduction & 1 \\
Cartilaginous involvement & 2 \\
Red or painful eyes & 1 \\
c-ANCA or PR3-ANCA & 5 \\
Eosinophil count $\geq 1\left(\times 10^{9} / \mathrm{L}\right)$ & -3 \\
Nodule, mass or cavitation on chest imaging & 2 \\
Granuloma on biopsy & 3 \\
GPA: total score of at least 5 & \\
Sensitivity: $90.7 \%$ and specificity: $93.5 \%$ & \\
\hline
\end{tabular}

ACR American College of Rheumatology, EULAR European League Against Rheumatism, GPA granulomatosis with polyangiitis, $c-A N C A$ cytoplasmic-antineutrophil cytoplasmic antibodies, PR3-ANCA ANCA against proteinase-3
Table 1 The ACR 1990 criteria for the classification of Wegener's granulomatosis (now known as GPA) [15]
1) Nasal or oral inflammation

Development of painful or painless oral ulcers or purulent or bloody nasal discharge

2) Abnormal chest radiograph

Chest radiograph showing the presence of nodules, fixed infiltrates, or cavities

3) Urinary sediment

Microhematuria ( $>5$ red blood cells per high power field) or red cell casts in urine sediment

4) Granulomatous inflammation on biopsy

Histologic changes showing granulomatous inflammation within the wall of an artery or in the perivascular or extravascular area (artery or arteriole)

Wegener's granulomatosis (GPA): at least 2 of 4 criteria are present Sensitivity: $88.2 \%$ and specificity: $92.0 \%$ 
$75 \mathrm{~dB}$, flat curves in tympanometry) lasting for 4-12 weeks. Only 2 patients reported aural complaints (discharge/pain) suggesting inflammatory cause of the disease. Otoscopic examination revealed thickening or retraction of the tympanic membrane while in one case it was reddened and another had visible scars on it with history of ear discharge. Due to the unclear clinical presentation and the radiological picture of mastoiditis in HRCT, suggesting otogenic cause of facial nerve paresis, all patients were qualified for surgical intervention. Procedures performed were the following: 3 cases-antromastoidectomy, 2 cases--canal wall up ear surgery (with partial decompression of facial nerve in one case), and 1 case--explorative tympanotomy. Intraoperative findings were similar in all patients: granulation tissue in tympanic cavity and/or pneumatic spaces of the mastoid process (Fig. 1). The lesions were partially or subtotally removed and sent for histopathologic assessment to exclude malignancy, specific, inflammatory, and autoimmunological diseases. Only $50 \%$ of results suggested vasculitis. In all patients, c-ANCA levels were evaluated: only one case had highly elevated level, four cases had slightly above normal limits, and one case had negative result at time of surgery but later turned positive after 2 months of follow-up period. In all cases, levels of ANCA against peroxidase 3 (PR3ANCA) were determined. Although, based on ACR criteria, the diagnosis of GPA was certain only in one patient from the study group, consultant rheumatologist recommended administration of ChT (cyclophosphamide) and steroids in all c-ANCA-positive cases. In one male patient, the course of the disease was fulminant, but his general state did not allow for such therapy. Two patients presented rapid progression of the disease, and died within 1 week and 2 months, respectively. Facial nerve palsy and mastoiditis were probably the revelators of generalized form of GPA in them. Four other patients manifested gradual improvement of hearing and facial nerve function after treatment. Six months after diagnosis of the disease, the ENT examination with audiometry of these four patients revealed HBI or HBII facial

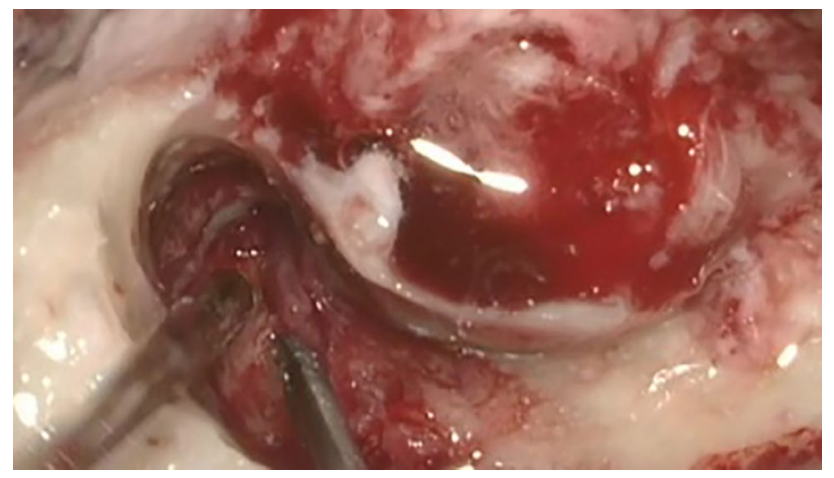

Fig. 1 Intraoperative findings in one of our patients (No 5): granulation tissue in aditus ad antrum and in the region of facial nerve nerve motoric action, and elevated pure tone audiometry curves for $20-35 \mathrm{~dB}$.

Detailed clinical data are given in Table 3.

\section{Discussion}

The authors present a unique series of 6 patients with facial nerve palsy and masked mastoiditis which constituted revelators of GPA. Although otological complaints are quite common in GPA patients, if isolated, they can be underdiagnosed and underestimated. Facial nerve palsy, a very pronounced symptom, is very rarely related to the GPA entity.

The American College of Rheumatology (ACR) 1990 criteria for the classification of (ANCA)-associated vasculitis [15] (Table 1) seem to be not specific and sensitive enough to state the diagnosis of GPA in localized forms of the disease. In 2017, a joint working group of Diagnostic and Classification Criteria for Primary Systemic Vasculitis (DCVAS) and collaborators, including the ACR, the European League Against Rheumatism (EULAR), and vasculitis foundations, proposed the ACR/EULAR 2017 provisional classification criteria for GPA [14] (Table 2). They have not been formally published yet, but their discriminative capacity was shown to be better in many studies [16]. In our group of patients, the new classification would allow for the confirmation of GPA in all analyzed patients. Based on ACR criteria, the diagnosis was certain only in one case with fulminant course of the disease, which was in fact generalized form although otologic manifestation was initially the most expressed and pronounced. It underlines the utility of new criteria in ENT patients with otological manifestation of the disease.

The differential assessment of facial nerve palsy is complex, and very often requires many consultations and examinations before the appropriate diagnosis is determined. In unilateral involvement, 70\% of cases are diagnosed as Bell's palsy [17]. In most cases of facial nerve palsy in the course of otitis media, surgical procedure is needed. In acute infections, myringotomy is usually the first step, followed by antromastoidectomy in patients without clinical improvement. In chronic diseases, urgent mastoidectomy with the removal of granulation tissue or cholesteatoma is almost always advocated [10]. In all of our patients, middle ear was opened and granulation tissue was found along facial nerve bony canal. Only in $50 \%$ of patients, the pathological diagnosis of GPA was reached. It is in line with data presented in the literature. Most authors suggest that biopsy, which according to recommendations is the basis to establish diagnosis, may not give a definite answer in cases of localized disease. Devaney et al. [18] reported that the typical GPA picture is visible in only $25-33 \%$ of specimen taken from the middle ear. Notwithstanding, biopsy of the granulation tissue enables exclusion of malignancy and other diseases, 


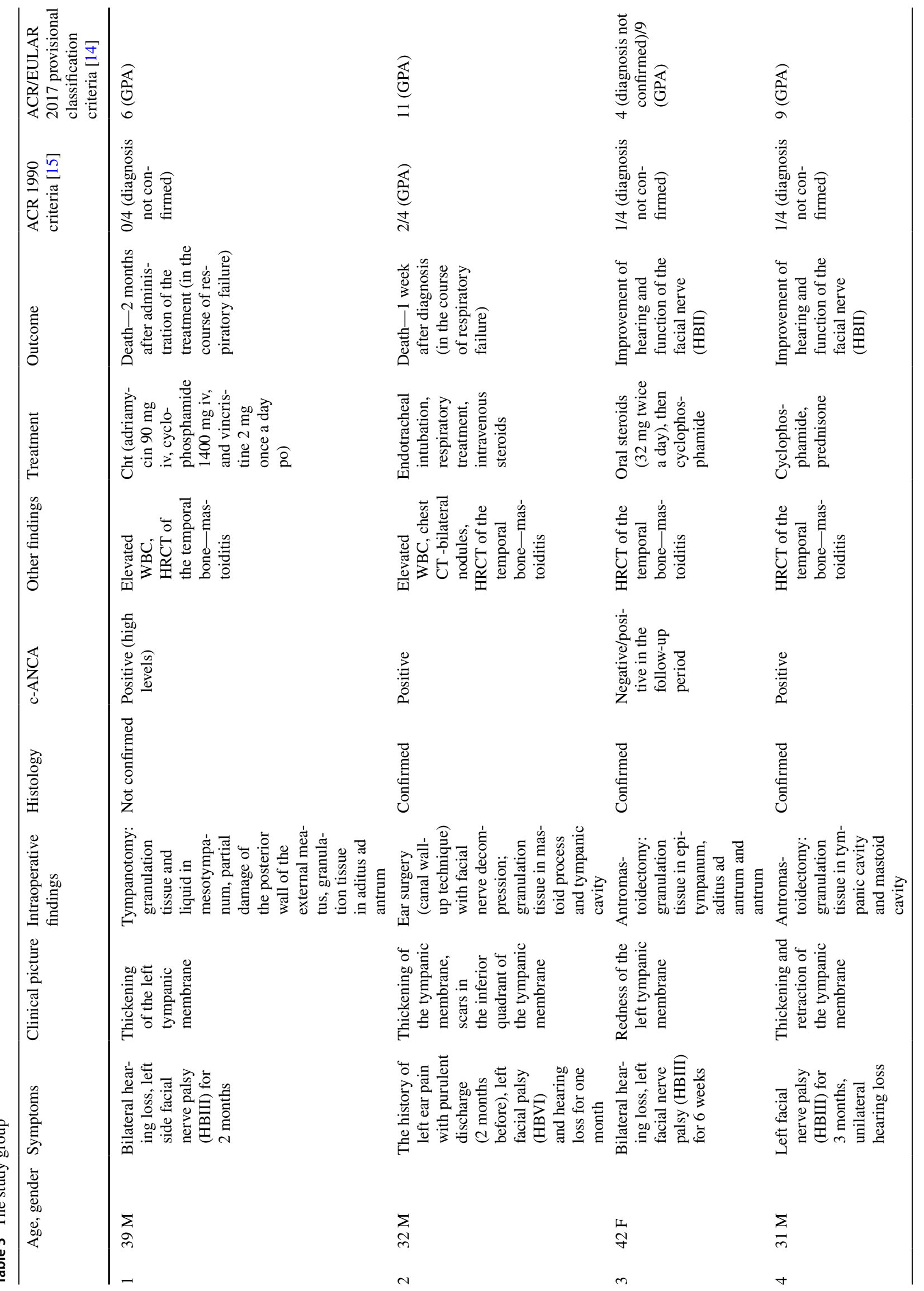




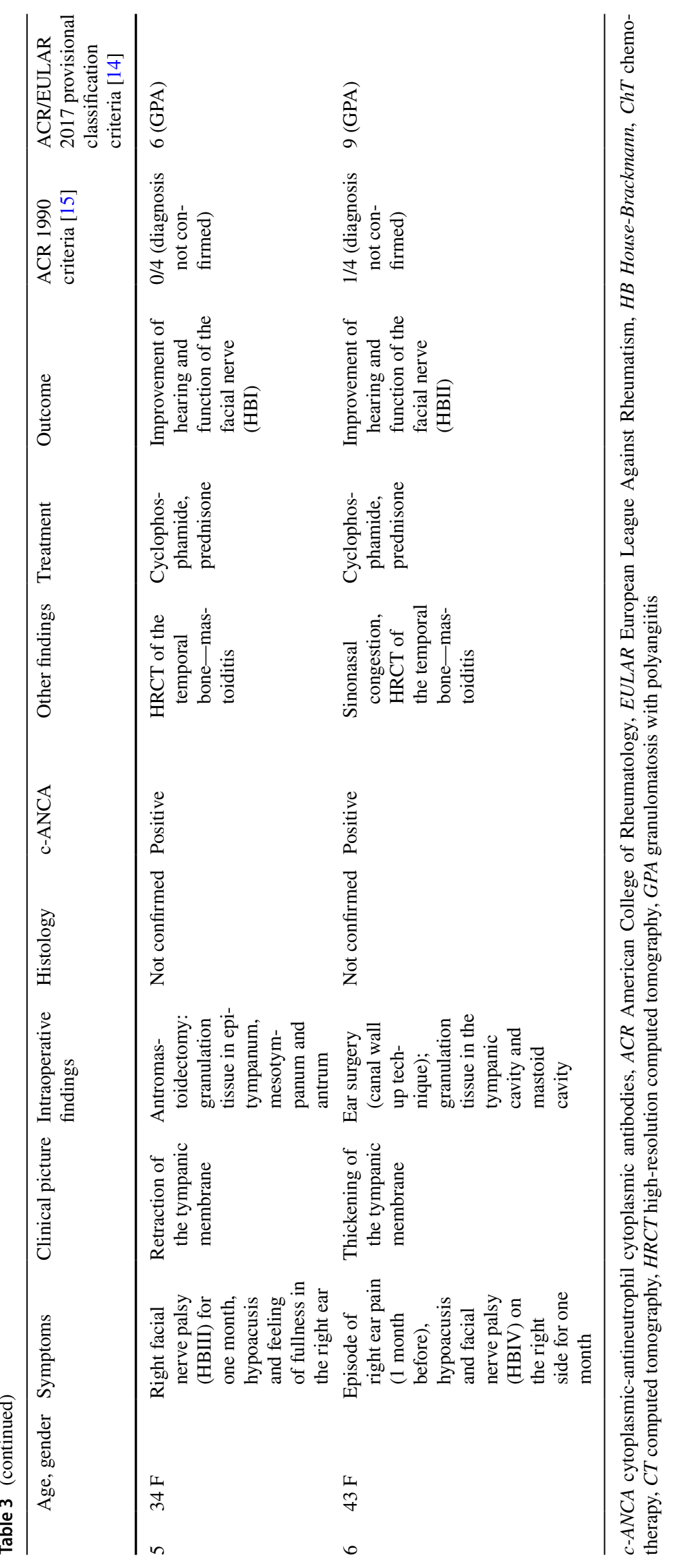


like TBC or sarcoidosis, which makes it very important as a part of differential diagnosis scheme. A higher percentage of true positive results is noted in biopsies of the nose and paranasal sinuses. Thus, if it is possible and indicated, such a diagnostic step should also be performed [19].

Cytoplasmic pattern antineutrophil cytoplasmic antibodies are highly specific for GPA, especially in its active phase. However, they are detected in only $22-25 \%$ of patients with localized GPA manifesting as a facial nerve palsy [5]. In addition, ANCA against proteinase (PR3) and antibodies against myeloperoxidase (MPO) are detected in $80 \%$ and $10 \%$ of cases with facial nerve involvement [4]. Among the six patients, only one case presented with high levels of c-ANCA, while four had slightly elevated levels. In addition, one case was negative for c-ANCA at time of surgery, but later became positive during the follow-up period. Such a scenario was also observed by other clinicians [20]. Repeated assessment of c-ANCA is highly recommended in not-obvious, underdiagnosed cases. In all patients, PR3ANCA were found which are also in line with literature data.

In most patients with GPA and facial nerve palsy, the paresis resolves after administration of the proper treatment [4]. Early initiation of adequate treatment tends to have better prognosis. Mur and al [8] presented a case of GPA with spontaneous improvement of facial nerve function after myringotomy and tube placement. In our patients, facial nerve palsy did not improve after surgery which prompted us for further diagnostics. Some authors underline that in active GPA, symptoms may even exacerbate after surgical procedure [21]. It is unclear whether it happens in the mechanism of "irritation" of "vasa nervorum" or progression of the disease [9]. Thus, it is highly recommended to avoid ear surgery in active disease if the diagnosis had been established. Based on the literature data and our own experience, it seems valuable to check c-ANCA levels in all patients with the clinical picture of masked mastoiditis and facial nerve palsy before surgical intervention. On the other hand, diagnostics should be expanded in all cases with atypical facial nerve palsy worsening or not improving after myringotomy or antromastoidectomy. The detailed evaluation should include MRI of the head and neck and laboratory tests to exclude viral (HIV, human herpesvirus 6, mumps virus, cytomegalovirus, and rubella virus), bacterial (Borrelia burgdorferi, Rickettsia, otogenic process), autoimmunological/metabolic (sarcoidosis, Sjogren's syndrome, sclerosis multiplex, GPA), and organic (tumors in the region of cerebellopontine angle, parotid gland, petrous bone, brainstem) pathologies [22, 23]. The elevated levels of c-ANCA could indicate further diagnostic steps and enable rapid administration of the adequate treatment. Both, extended diagnostics and targeted therapy, are conducted by rheumatologists.

Nowadays, treatment of GPA involves intensive immunosuppressive therapy with high doses of corticosteroids, along with cyclophosphamide or rituximab. In severe cases, the combination of all of them is possible [24]. Once remission is achieved, patients are administered less toxic immunosuppression, such as azathioprine and low dose glucocorticosteroid [25]. Even localized disease could be very aggressive, and may lead to high morbidity [25]. It is widely discussed in the literature if toxic treatment should be administered when the diagnosis of GPA is not confirmed histologically. Many authors underline that the delay in the initiation of the treatment may negatively affect the prognosis, and thus, it should be started when c-ANCA levels are elevated and clinical picture strongly suggests GPA [19]. Truly, such cases should be diagnosed with GPA according to the new ACR/EULAR 2017 provisional classification criteria. In our group, the treatment was administered when GPA was identified in biopsy specimen or c-ANCA serologic tests were positive. Two patients presented rapid progression of the disease although the proper treatment was administered, possibly due to the cytotoxicity of the immunosuppressants, exacerbation of the disease after surgical procedure, or delayed accurate diagnosis. However, we also proved that systemic drugs allow for the improvement of hearing and function of the facial nerve in localized disease-such results were observed in all of our patients.

In the literature of the last 20 years, we have not found the descriptions of GPA cases manifesting as facial palsy and masked mastoiditis. There are several articles presenting patients with unilateral or bilateral paresis of the VIIth nerve, in most cases presenting as a sole initial symptom or as a component or complication of acute of chronic otitis media [26-28]. A wide and interesting review of such cases is presented by Iannella et al. [4]. Our study group consists of patients in whom facial nerve palsy was the first and the most pronounced symptom that brought them to the physician. Although ENT examination did not reveal emerging otoscopic changes, detailed history showed otologic origin of the paresis, and thus prompted for surgical intervention. In all cases, granulation tissue was found in middle ear spaces, and of these cases, half was identified as typical for GPA. Finally, all patients presented with positive c-ANCA. It cannot be ruled out that the prior assessment of c-ANCA could protect 5 out of 6 patients from surgical intervention. In our study group, four cases were truly localized forms of the disease and had good treatment response, which was observed as improvement of hearing and function of the VIIth nerve. In two patients, facial nerve palsy and masked mastoiditis were revelators of generalized form of GPA.

\section{Conclusions}

GPA is an insidious and multi-symptomatic disease. It should be included into differential diagnosis in all cases of persistent facial nerve palsy especially when otological 
symptoms coexist. In patients with no changes in otoscopy, HRCT of temporal bone should be considered. When masked mastoiditis is suspected, c-ANCA titer should be assessed before surgical intervention. If negative, middle ear should be inspected and if granulation tissue is present, the lesion should be examined histopathologically. Even localized GPA could be very aggressive, revelating generalized form of the disease. Rapid systemic treatment of GPA can protect hearing and facial nerve from permanent severe dysfunction. The ACR/EULAR 2017 provisional classification criteria for GPA seem to be valuable tool in diagnosing ENT patients with localized otological form of the disease.

Author contributions All authors whose names appear on the submission. (1) Made substantial contributions to the conception or design of the work; or the acquisition, analysis, or interpretation of data; or the creation of new software used in the work; (2) Drafted the work or revised it critically for important intellectual content; (3) Approved the version to be published; and (4) Agree to be accountable for all aspects of the work in ensuring that questions related to the accuracy or integrity of any part of the work are appropriately investigated and resolved. MJ, BA, and WM contributed to conceptualization; MJ performed formal analysis and investigation; $\mathrm{MJ}$ and $\mathrm{BA}$ were involved in methodology and Writing - original draft preparation; YR and WM contributed to writing - review and editing; WM supervised this study.

Funding No funding was received for conducting this study.

Data availability Yes.

Code availability MS Excel.

\section{Declarations}

Conflict of interest The authors have no relevant financial or non-financial interests to disclose.

Ethical approval Approval was obtained from the ethics committee of Poznan University of Medical Sciences. The procedures used in this study adhere to the tenets of the Declaration of Helsinki.

Consent to participate Informed consent was obtained from all individual participants included in this study.

Consent for publication Patients signed informed consent regarding publishing their data and photographs.

Open Access This article is licensed under a Creative Commons Attribution 4.0 International License, which permits use, sharing, adaptation, distribution and reproduction in any medium or format, as long as you give appropriate credit to the original author(s) and the source, provide a link to the Creative Commons licence, and indicate if changes were made. The images or other third party material in this article are included in the article's Creative Commons licence, unless indicated otherwise in a credit line to the material. If material is not included in the article's Creative Commons licence and your intended use is not permitted by statutory regulation or exceeds the permitted use, you will need to obtain permission directly from the copyright holder. To view a copy of this licence, visit http://creativecommons.org/licenses/by/4.0/.

\section{References}

1. Martinez Del Pero M, Rasmussen N, Chaudhry A, Jani P, Jayne D (2013) Structured clinical assessment of the ear, nose and throat in patients with granulomatosis with polyangiitis (Wegener's). Eur Arch Otorhinolaryngol 270(1):345-354. https://doi.org/10.1007/ s00405-012-2110-8

2. Kempf HG (1989) Ear involvement in Wegener's granulomatosis. Clin Otolaryngol Allied Sci 14(5):451-6. https://doi.org/10. 1111/j.1365-2273.1989.tb00403.x

3. Wierzbicka M, Szyfter W, Puszczewicz M, Borucki L, Bartochowska A (2011) Otologic symptoms as initial manifestation of Wegener granulomatosis: diagnostic dilemma. Otol Neurotol 32(6):996-1000. https://doi.org/10.1097/MAO.0b013e3182 2558fd

4. Iannella G, Greco A, Granata G, Manno A, Pasquariello B, Angeletti D, Didona D, Magliulo G (2016) Granulomatosis with polyangiitis and facial palsy: literature review and insight in the autoimmune pathogenesis. Autoimmun Rev 15(7):621-631. https:// doi.org/10.1016/j.autrev.2016.02.005

5. Yoshida N, Iino Y (2014) Pathogenesis and diagnosis of otitis media with ANCA-associated vasculitis. Allergol Int 63(4):523532. https://doi.org/10.2332/allergolint.14-RAI-0774

6. Debski MG, Zycińska K, Czarkowski M, Zukowska M, Wardyn KA, Bar-Andziak E (2007) Postepujaca utrata słuchu jako wiodacy objaw ziarniniakowatości Wegenera [Progressive hearing loss as the leading sign of Wegener's granulomatosis]. Pol Arch Med 117(5-6):266-9

7. Yoshida N, Hara M, Hasegawa M, Matsuzawa S, Shinnabe A, Kanazawa H, Iino Y (2014) Reversible cochlear function with ANCA-associated vasculitis initially diagnosed by otologic symptoms. Otol Neurotol 35(1):114-120. https://doi.org/10.1097/ MAO.0000000000000175

8. Mur T, Ghraib M, Khurana JS, Roehm PC (2019) Granulomatosis with polyangiitis presenting with bilateral hearing loss and facial paresis. OTO Open. https://doi.org/10.1177/2473974X18818791

9. Takagi D, Nakamaru Y, Maguchi S, Furuta Y, Fukuda S (2002) Otologic manifestations of Wegener's granulomatosis. Laryngoscope 112(9):1684-1690. https://doi.org/10.1097/00005537200209000-00029

10. Mansour S, Magnan J, Nicolas K, Haidar H (2018) Middle ear diseases. Springer. https://doi.org/10.1007/978-3-319-72962-6_2

11. Voudouris C, Psarommatis I, Nikas I, Kafouris D, Chrysouli K (2015) Pediatric masked mastoiditis associated with multiple intracranial complications. Case Rep Otolaryngol 2015:897239. https://doi.org/10.1155/2015C

12. Joseph EM, Sperling NM (1998) Facial nerve paralysis in acute otitis media: cause and management revisited. Otolaryngol Head Neck Surg 118(5):694-696. https://doi.org/10.1177/0194599898 11800525

13. Santos F, Salviz M, Domond H, Nadol JB (2015) Otopathology of vasculitis in granulomatosis with polyangitis. Otol Neurotol 36(10):1657-1662. https://doi.org/10.1097/MAO.0000000000 000868

14. Yoo J, Kim HJ, Ahn SS, Jung SM, Song JJ, Park YB, Lee SW (2018) The utility of the ACR/EULAR 2017 provisional classification criteria for granulomatosis with polyangiitis in Korean patients with antineutrophil cytoplasmic antibody-associated vasculitis. Clin Exp Rheumatol 36(Suppl 111):85-87

15. Leavitt RY, Fauci AS, Bloch DA, Michel BA, Hunder GG, Arend WP, Calabrese LH, Fries JF, Lie JT, Lightfoot RW Jr et al (1990) The American College of rheumatology 1990 criteria for the classification of Wegener's granulomatosis. Arthritis Rheum 33(8):1101-1107. https://doi.org/10.1002/art.1780330807 
16. Yoo J, Kim HJ, Ahn SS, Jung SM, Song JJ, Park YB, Lee SW (2018) The utility of the ACR/EULAR 2017 provisional classification criteria for granulomatosis with polyangiitis in Korean patients with antineutrophil cytoplasmic antibody-associated vasculitis. Clin Exp Rheumatol 111(2):85-87

17. Pothiawala $S$, Lateef $F$ (2012) Bilateral facial nerve palsy: a diagnostic dilemma. Case Rep Emerg Med 2012:458371. https://doi. org/10.1155/2012/458371

18. Devaney KO, Travis WD, Hoffman G, Leavitt R, Lebovics R, Fauci AS (1990) Interpretation of head and neck biopsies in Wegener's granulomatosis. A pathologic study of 126 biopsies in 70 patients. Am J Surg Pathol 14(6):555-64. https://doi.org/10. 1097/00000478-199006000-00006

19. Knopf A, Chaker A, Stark T, Hofauer B, Lahmer T, Thürmel K, Bas M (2015) Clinical aspects of granulomatosis with polyangiitis affecting the head and neck. Eur Arch Otorhinolaryngol 272(1):185-193. https://doi.org/10.1007/s00405-014-2973-y

20. Bibas A, Fahy C, Sneddon L, Bowdler D (2001) Facial paralysis in Wegener's granulomatosis of the middle ear. J Laryngol Otol 115(4):304-306. https://doi.org/10.1258/0022215011907253

21. Fauci AS, Haynes BF, Katz P, Wolff SM (1983) Wegener's granulomatosis: prospective clinical and therapeutic experience with 85 patients for 21 years. Ann Intern Med 98(1):76-85. https://doi.org/ 10.7326/0003-4819-98-1-76

22. Finsterer J (2008) Management of peripheral facial nerve palsy. Eur Arch Otorhinolaryngol 265(7):743-752. https://doi.org/10. 1007/s00405-008-0646-4

23. Heckmann JG, Urban PP, Pitz S, Guntinas-Lichius O, Gágyor I (2019) The diagnosis and treatment of idiopathic facial paresis (Bell's Palsy). Dtsch Arztebl Int 116(41):692-702. https://doi.org/ 10.3238/arztebl.2019.0692
24. Jones RB, Tervaert JW, Hauser T, Luqmani R, Morgan MD, Peh CA, Savage CO, Segelmark M, Tesar V, van Paassen P, Walsh D, Walsh M, Westman K, Jayne DR, European Vasculitis Study Group (2010) Rituximab versus cyclophosphamide in ANCAassociated renal vasculitis. N Engl J Med 363(3):211-20. https:// doi.org/10.1056/NEJMoa0909169

25. Peters JE, Gupta V, Saeed IT, Offiah C, Jawad ASM (2018) Severe localised granulomatosis with polyangiitis (Wegener's granulomatosis) manifesting with extensive cranial nerve palsies and cranial diabetes insipidus: a case report and literature review. BMC Neurol 18(1):59. https://doi.org/10.1186/s12883-018-1058-8

26. Ono N, Yoshihiro K, Oryoji D, Matsuda M, Ueki Y, Uezono S, Kai Y, Himeji D, Niiro H, Ueda A (2013) Four cases of MPO-ANCA-positive vasculitis with otitis media, and review of the literature. Mod Rheumatol 23(3):554-563. https://doi.org/10. 1007/s10165-012-0682-1

27. Kukushev G, Kalinova D, Sheytanov I, Rashkov R (2017) Two clinical cases of granulomatosis with polyangiitis with isolated otitis media and mastoiditis. Reumatologia 55(5):256-260. https:// doi.org/10.5114/reum.2017.71643 (Epub 2017 Oct 28)

28. Qaisar H, Shenouda M, Shariff M, Cheema A, Tang X, Kaplan A (2019) Granulomatosis with polyangiitis manifesting as refractory otitis media and mastoiditis. Arch Iran Med 22(7):410-413

Publisher's Note Springer Nature remains neutral with regard to jurisdictional claims in published maps and institutional affiliations. 